\title{
Dynamic propensity in a kinetically constrained lattice gas
}

\author{
Lester O. Hedges and Juan P. Garrahan \\ School of Physics and Astronomy, University of Nottingham, Nottingham, NG7 2RD, \\ UK
}

\begin{abstract}
We apply the concept of dynamic propensity to a simple kinetically constrained model of glass formers, the two-vacancy assisted triangular lattice gas, or (2)-TLG. We find that the propensity field, defined in our case as the local rootmean square displacement averaged over the ensemble of trajectories with identical initial configurations, is a good measure of dynamical heterogeneity. This suggests a configurational origin for spatial fluctuations of the dynamics, but just as in the case of atomistic systems, we find that propensity is not correlated to any simple structural property. We show instead that certain extended clusters of particles connected to vacancies correlate well with propensity, indicating that these are the fundamental excitations of the (2)-TLG. We also discuss time-correlations and the correlation between configurations within the propensity ensemble.
\end{abstract}

\section{Introduction}

On approach to their glass transition [1] glass forming systems display increasingly heterogeneous dynamics [2]. This dynamic heterogeneity is not correlated in any obvious way to structural features. In order to uncover a possible configurational origin for dynamic heterogeneity Harrowell and coworkers recently proposed the concept of dynamic propensity [3, 4]: a particle's propensity is defined as some measure of its mobility over a period of time, such as its mean square displacement, averaged over all dynamic trajectories which start from the same initial configuration. This ensemble of trajectories is sometimes referred to as the iso-configurational ensemble. Dynamic propensity was found to be a good indicator of dynamic heterogeneity in simulations of atomistic models [3] for times at least as long as the structural relaxation time. While this suggested a configurational origin for the heterogeneity in the dynamics, no correlation was found between dynamic propensity and simple structural measures such as local free volume or local composition [3].

In this paper we apply the idea of dynamic propensity to a kinetically constrained model (KCM) of a glass former, the two-vacancy assisted triangular lattice gas [5], or (2)-TLG. We show that the heterogeneity in the dynamics can be described by an appropriately defined propensity field. We find that propensity is not correlated to simple local configurational properties such as local density. Instead we show that 


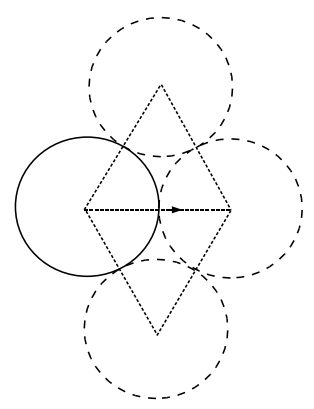

Figure 1. The kinetic constraint for nearest neighbour particle jumps in the twovacancy assisted triangular lattice gas. Dashed lines refer to unoccupied sites.

a non-local structural property, the connectedness of particles to clusters of vacancies, serves as a good predictor of dynamic propensity. We also discuss the behaviour of crosscorrelations between configurations in the iso-configurational ensemble and its relation to the dynamic phase separation associated with dynamic heterogeneity.

\section{2. (2)-TLG model and propensity}

We consider the lattice gas model introduced by Jäckle and Krönig [5]. This model is itself a variant of the constrained lattice models proposed by Kob and Andersen [6, 7, 8, 9]. It consists of a set of particles that move on a two-dimensional lattice of triangular geometry. There are no static correlations between particles and at most each site can only hold one particle at a time. Any particle on the lattice can only move to one of its six nearest neighbour sites if the following rules are satisfied: (i) the target site is unoccupied and (ii) both the two mutual nearest neighbours of the initial and target site are also empty, see Fig. 1. The model is often referred to as that of two-vacancy assisted hopping on the triangular lattice, or more simply the (2)-TLG. The absence of any static correlations allows initial configurations to be constructed by randomly placing particles into the lattice until the desired density is reached. Although not

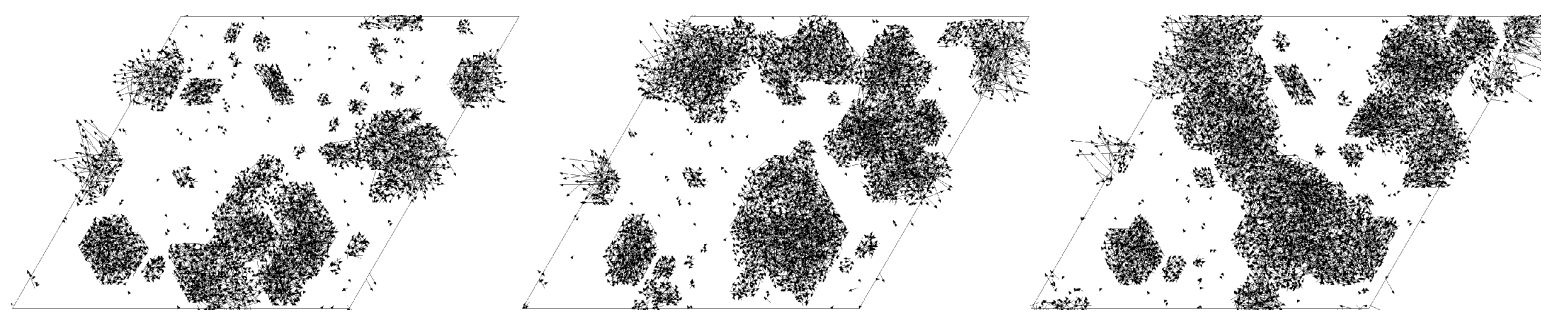

Figure 2. Three particle trajectories from the same equilibrium configuration at $\rho=0.79$. Particle displacements are shown as arrows joining the initial and final positions. Each trajectory is $t=10^{6}$ Monte Carlo sweeps in length and differs only in the random sequence of attempted particle moves. 

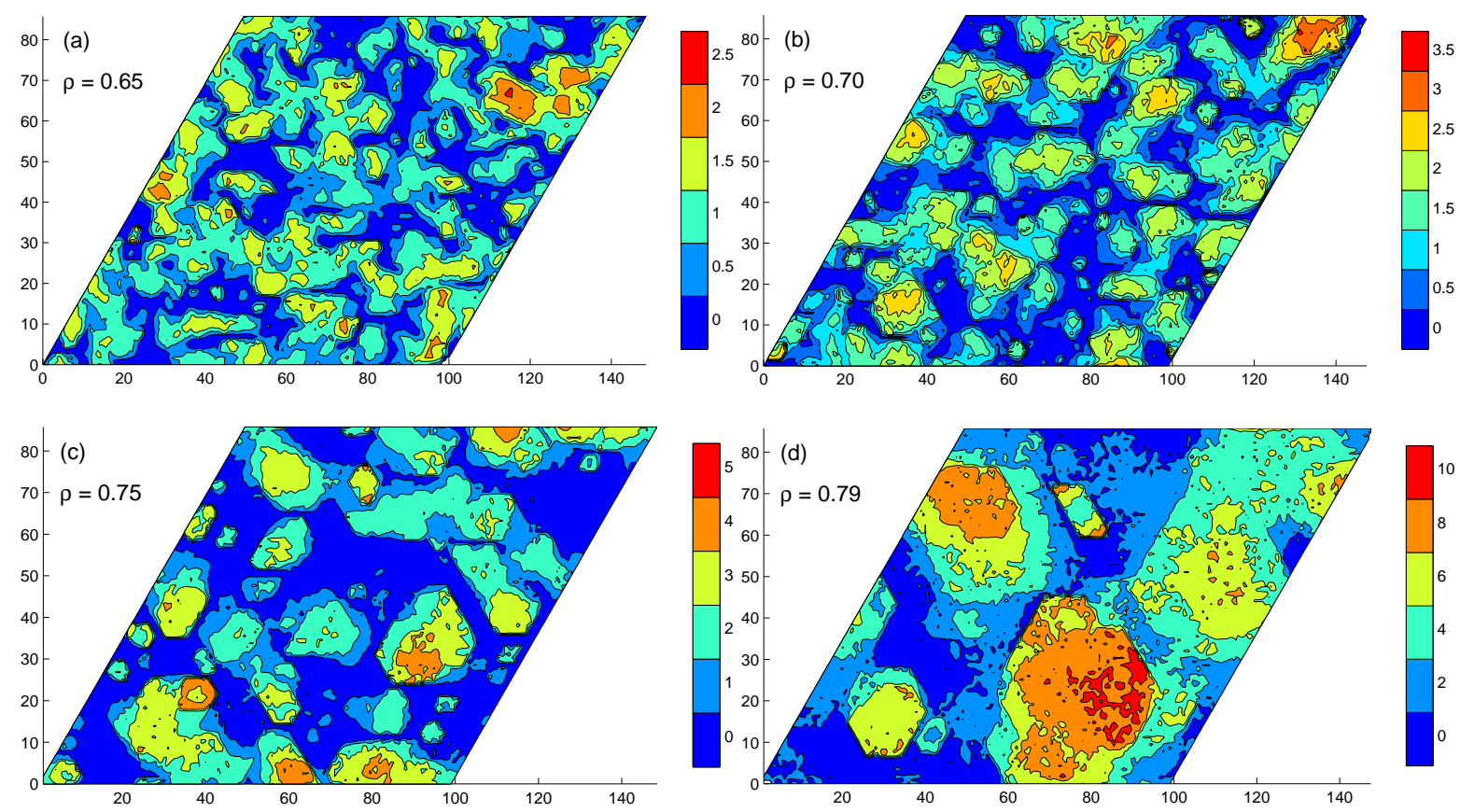

Figure 3. Propensity maps for the (2)-TLG at four different particle densities, $\rho=0.65$ (a), $\rho=0.7$ (b), $\rho=0.75$ (c) and $\rho=0.79$ (d). Each map has been created by averaging over 100 independent trajectories.

intended to represent a physical system the dynamical rules can be interpreted as the steric constraint on particle motion within a dense fluid. For increasing particle density the model shows a rapid dynamical slowdown and clear dynamical heterogeneity [10]. The dynamics of the model are indicative of a fragile glass former [10].

For our model we choose to define the propensity of particle $i$ as its root-mean squared displacement, $\sqrt{\left\langle\Delta r_{i}{ }^{2}\right\rangle}$. With this choice propensity has units of length (rather than length squared as in [3]). The average is over all trajectories starting from the same initial configuration using lattice gas Monte Carlo (MC) dynamics (or continuous time $\mathrm{MC}$ for high densities [11]). That is, for a given initial condition our propensity ensemble is that of all possible randomly attempted particle moves. This ensures that the dynamics of the system has opportunity to proceed via a different route during each run: Fig. 2 shows the resulting particle displacements for three different trajectories starting from the same $\rho=0.79$ initial configuration. The propensity ensemble as defined here is the analog in the MC case to that of randomized momenta used in MD simulations [3].

Fig. 3 shows the spatial distribution of propensity at four different particle densities $\rho$. The times for each density is the relaxation time $\tau_{\alpha}(\rho)$ extracted from the persistence function [10]. The data is represented as a contour plot. Each propensity map has been averaged over 100 independent trajectories with a lattice size of $100 \times 100$ sites. The relaxation time at $\rho=0.79$ is approximately $10^{4}$ times larger than of the lowest density shown, $\rho=0.65$. A comparison between the four panels shows the distribution of propensity becoming increasingly spatially heterogeneous as the density 


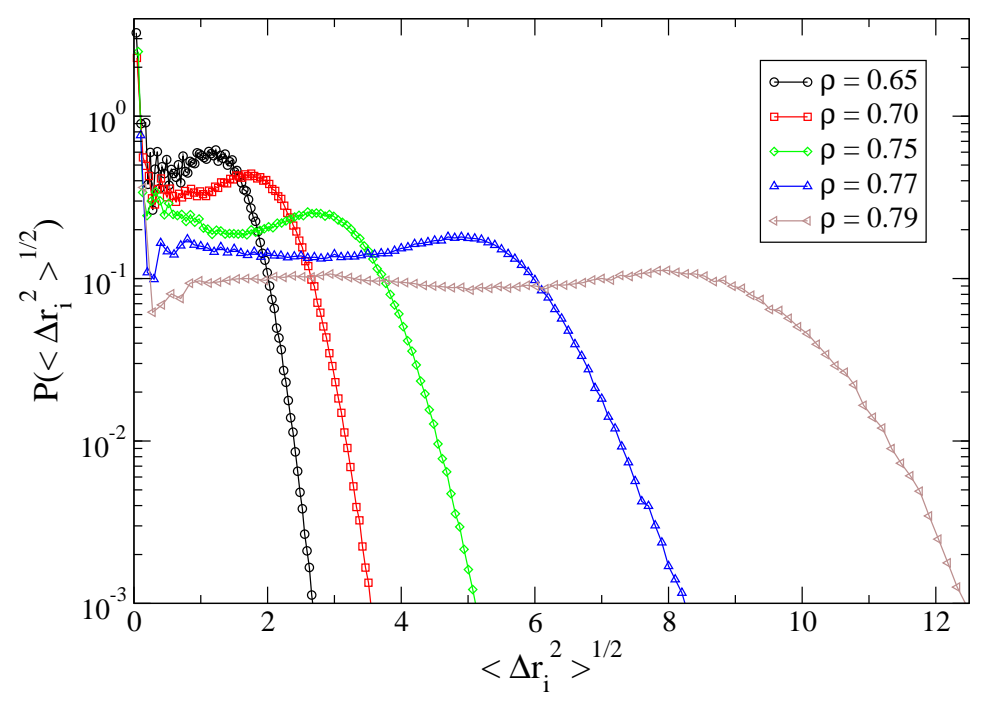

Figure 4. Distribution of propensities at various particle densities $\rho$ and times $\tau_{\alpha}(\rho)$.

is increased. High propensity regions become more localised in nature whilst domains of low propensity are observed to grow in size. This is similar to what is observed in atomistic systems as temperature is decreased [3].

It is also informative to analyse the distribution of particle propensities as a function of density, Fig. 4. This is analogous to the van Hove function. The figure shows that the distribution widens significantly for increasing particle density. Interestingly, at high density the distributions appear remarkably flat over a large range of propensities. This suggests that for these time scales, the curve is not well fit by two Gaussian profiles (or a delta and a Gaussian), one describing the dynamics of low propensity particles, the other characteristic of high propensity behaviour. As argued in Ref. [12], this is a sensible approximation at times either much smaller or much larger than $\tau_{\alpha}$. For the times comparable to $\tau_{\alpha}$ shown here the overall distribution appears to be that of a convolution of Gaussian functions corresponding to particles which began diffusing at all times between 0 and $t$ [12]. This seems to be an important feature of transport decoupling which deserves further exploration.

\section{Linking structure and dynamics}

We next try to ascertain what aspect of the initial structure, if any, is responsible for the heterogeneity observed in the spatial distribution of propensity. As a starting point it is useful to first consider a simple local property of the system such as the local density. In any instantaneous configuration the ability of a particle in the (2)-TLG to make a single step is entirely determined by its local neighbourhood as this governs the dynamic rules of the system. Those particles which have a higher number of vacancies amongst their 


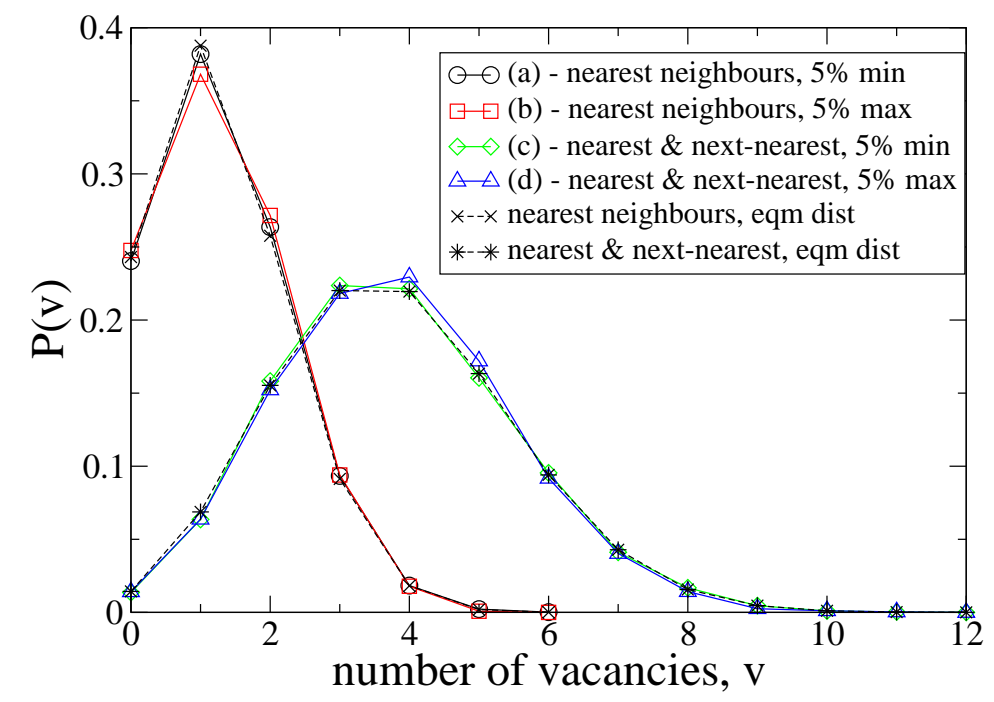

Figure 5. Distribution of vacancies amongst, nearest neighbours for particles within the lowest $5 \%$ propensity (a), nearest neighbours for particles within the highest $5 \%$ propensity (b), nearest and next-nearest neighbours for particles within the lowest $5 \%$ propensity (c), and nearest and next-nearest neighbours for particles within the highest $5 \%$ propensity (d). The figure shows that these distributions coincide with those of a random environment at this density.

nearest neighbours will be more likely to move. If local density does correlate well then one would expect the local neighbourhood of high propensity particles to be markedly different from those for which the propensity is low.

Fig. 5 shows the distribution of vacancies amongst nearest neighbours for particles within the lowest $5 \%$ propensity, nearest neighbours for particles within the highest $5 \%$ propensity, nearest and next-nearest neighbours for particles within the lowest $5 \%$ propensity, nearest and next-nearest neighbours for particles within the highest $5 \%$ propensity. It is clear that there is nothing special about the local environment of the highest propensity particles, as the distribution of vacancies appears to exactly match the low propensity curve, even when coarse-grained over several particle distances. This is again analogous to what was found in atomistic simulations [3]. In a system like the (2)-TLG, for which the dynamics are highly collective, many cooperative rearrangements are required in order for a particle to undergo a significant displacement. As such it is perhaps unsurprising that local density fails to provide a suitable prediction of the long time propensity for motion. In order to provide a more accurate prediction of the spatial distribution one needs to consider some non-local feature of the initial structure. Interestingly, in the less cooperative (1)-TLG [10], where dynamics proceeds through diffusion of vacancy pairs, we find a similar lack of correlation between propensity and structure as that given by Fig. 5.

It is not individual vacancies but rather clusters of vacancies that help facilitate the 

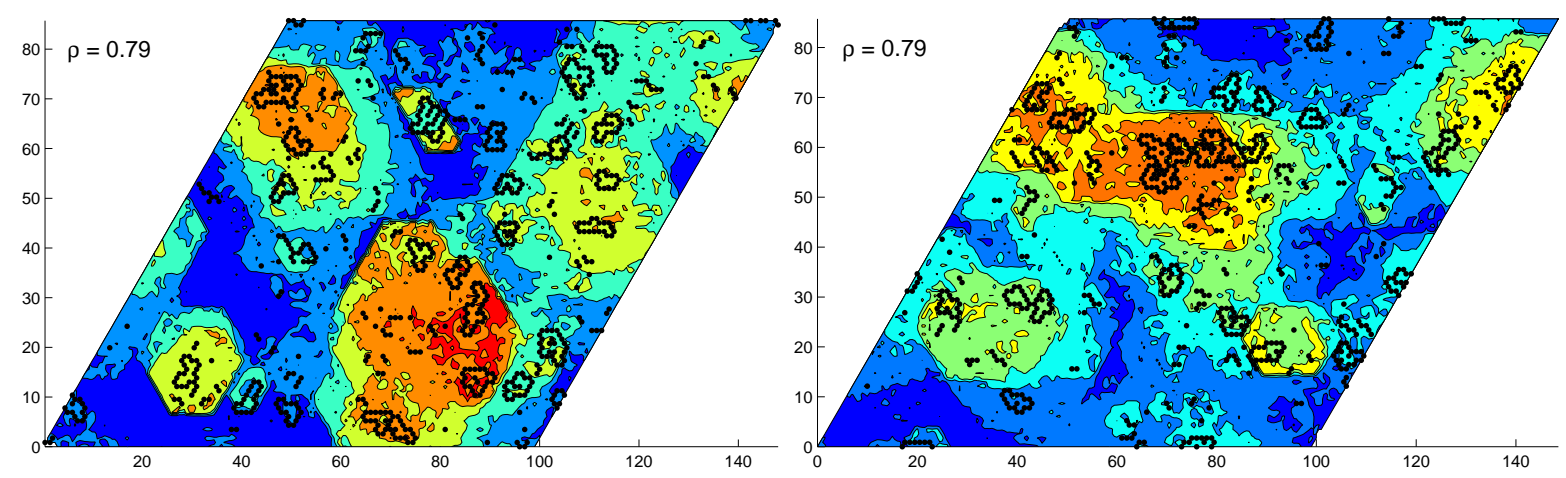

Figure 6. Two propensity maps and clusters of particles with high connectivity at density $\rho=0.79$. The first map is the same as that of Fig. 3(d).
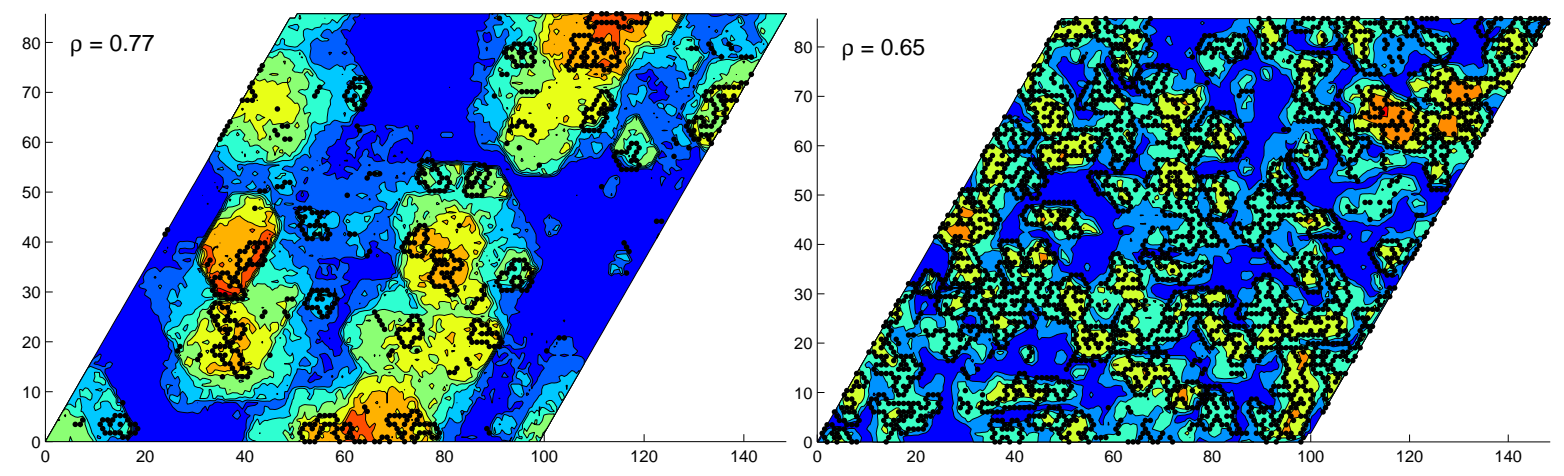

Figure 7. Same as previous figure, but now at two lower densities.

rearrangement of particles in some collective manner [7, 9, 10]. Considering this aspect of the dynamics we choose to classify particles in the initial configuration according to the size of vacancy clusters to which they are immediately connected. For example, although a particle may only have a single vacancy amongst its nearest neighbours this vacancy could itself be part of a cluster of appreciable size. A feature of this nature would not be picked up by any localised measure. Using a Hoshen-Kopelman algorithm [13] we identify and label all distinct vacancy clusters within a given initial configuration. Following the labeling procedure it is trivial to determine the size of each cluster. Each particle is then assigned a value equal to the sum of the vacancy cluster sizes to which it is connected. We define this value as the cluster connectivity.

Fig. 6] shows two propensity maps at the highest density we studied, $\rho=0.79$. Highlighted in each panel (black circles) are those particles whose cluster connectivities are above a chosen threshold (highest $10 \%$ in this case). These particles typically form closed rings around a large vacancy cluster. It is clear that the most highly connected particles correlate extremely well with those particles showing the highest propensity. This is not only true for the highest density configurations, for which the propensity distribution is most heterogeneous, a good agreement is also observed at lower density. In Fig. 7 we show the same for densities $\rho=0.77$ and $\rho=0.65$ (the threshold for $\rho=0.77$ is $10 \%$, and for $\rho=0.65$ is $50 \%$ ). For the lower density of $\rho=0.65$ the 


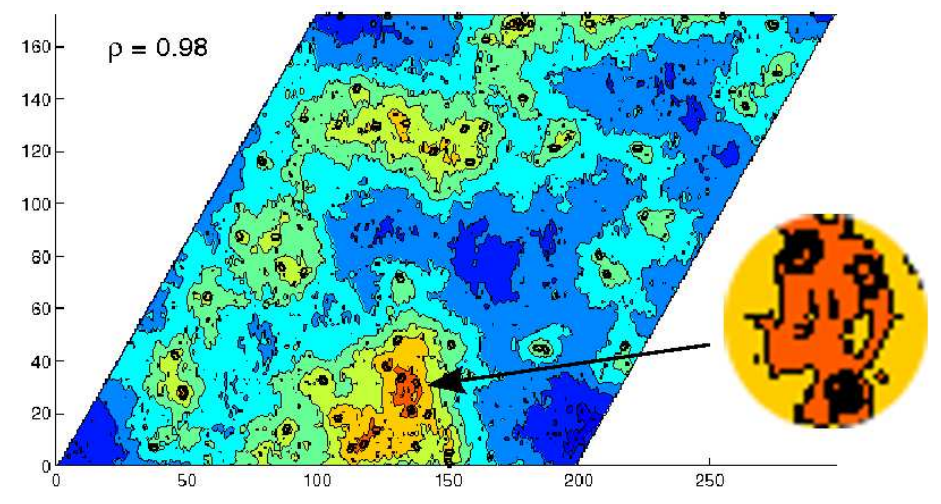

Figure 8. Propensity map and clusters of particles with high connectivity in the (1)-TLG at density $\rho=0.98$.

high propensity regions can almost be entirely described by the particles whose cluster connectivity falls into the top $50 \%$ of the system.

In Fig. 8 we show a similar propensity map for the (1)-TLG at a high density of particles. While less striking, the behaviour is analogous to that of the (2)-TLG: the propensity is heterogeneously distributed in space, and regions of high propensity correlate with highly connected clusters.

The result above is also apparent when analysing the distribution of propensity for particles with the minimum and maximum cluster connectivity, as shown by Fig. 9 for the (2)-TLG. In contrast to the local density measure, the two distributions are clearly different, the particles with minimum connectivity showing a peak at low propensity whereas the peak is shifted to higher propensity for particles with high connectivity.

Our results here are another indication of the difficulty in trying to connect dynamics to structure in atomistic systems. Even in the simple systems we consider, like the (2)-TLG and (1)-TLG, one has to work pretty hard to obtain any significant correlation between a structural measure and the observed dynamics, and the success has much to do with the detailed prior knowledge of the dynamics in these models. Devising a similar cluster scheme which is of any use in atomistic models appears to be a very complicated task.

\section{Time dependence of propensity}

An important issue to understand is the time dependence of the propensity. Fig. 10 shows three propensity maps created using an initial configuration of $100 \times 100$ sites at density $\rho=0.79$. The three panels show the distribution of propensity at different time scales, (a) $t=20$, (b) $t=500$ and (c) $t=10^{4}$. Here we do not use a contour plot but instead construct the distribution from circles centered about a particle's position in the initial configuration with a radius equal to its propensity. Filled circles indicate the particles whose cluster connectivity is within the top $5 \%$ of the system.

Comparing the figures it is evident that the highly connected particles act as seeds 

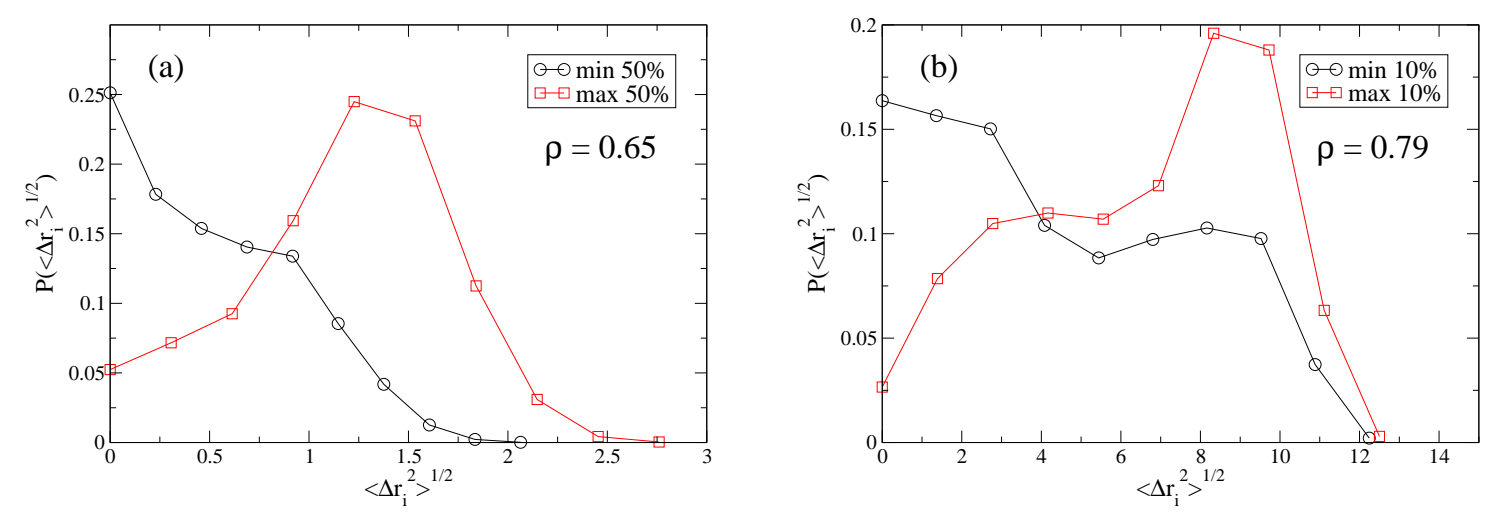

Figure 9. Distribution of propensity for particles which are connected to the largest and smallest vacancy clusters.

for mobility within the system with propensity circles seen to emanate outwards from these hot spots. In this sense, propensity at shorter times is a good predictor of propensity at longer times. While clearly in our system there is no such thing as a local Debye-Waller factor, this spatial correlation between short and long time dynamics does resemble the one found in [4]. As the system evolves some interesting features can be seen in the propensity maps. In cases where two or more large vacancy clusters are in close proximity one may observe the individual clusters to coalesce. This is often accompanied by a sudden burst of motion at the contact point as indicated by a region of newly mobile small circles. The propensity of these particles may then rapidly grow as motion persists in the region. This behaviour is consistent with the shape of the propensity distributions shown in Fig. 4.

Examining the evolution of the propensity field sheds light on the nature of the (2)TLG dynamics. As suggested earlier it is the clusters of vacancies that help facilitate the cooperative rearrangement of particles within the system. From this we find highly connected particles which themselves form clusters that move, coalescing and fragmenting into clusters of different shape and size. It appears that these clusters underly the dynamics and are in effect the essential excitations for the model. The initial configuration represents only a single instantaneous indication of the system's structure. By labeling particles according to their cluster connectivity we only manage to capture the hot spots at a particular moment in time. By analysing the evolution of the most highly connected particles we should be able to track the motion of the excitations throughout the lattice. Note this is not the same as simply looking at the motion of vacancies.

The motion of one of the clusters is illustrated in Fig. 11. Here we show the position of the particles with the top $5 \%$ connectivity at three different times. Highlighted in the three panels is the motion of two nearby clusters, which are seen to come together and eventually coalesce. Fig. 11] shows two characteristic lengthscales, the typical cluster size 
Dynamic propensity in a kinetically constrained lattice gas

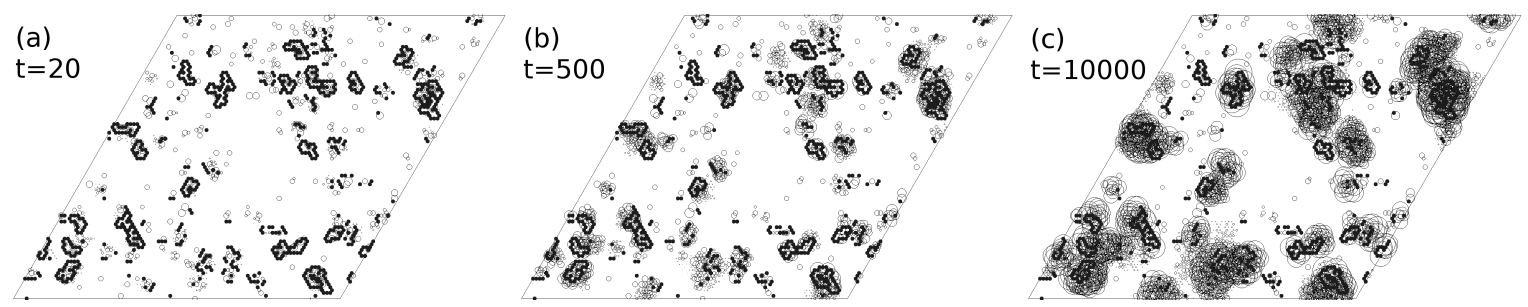

Figure 10. Time evolution of the propensity map [14].

and the typical distance between them. This resembles the super-defect scenario of [8]. One difference however, at least superficially, appears to be the dynamics: super-defects are supposed to be free to propagate; the clusters we find have pinned end-points, they fluctuate and may combine to form larger clusters, but are not free to move away. In any case, it would be worth exploring the connection to super-defects further.

\section{Propensity correlations and time-correlations}

We end by considering time correlations and cross-correlations or overlaps within the propensity ensemble. The normalized autocorrelation function is $C(t) \equiv$ $\left\langle n_{i}(0) n_{i}(t)\right\rangle_{\mathrm{c}} /\left\langle n_{i}(0) n_{i}(0)\right\rangle_{\mathrm{c}}$, where $n_{i}=\{0 ; 1\}$ specifies the site occupancy, and $\langle\cdot\rangle_{\mathrm{c}}$ indicates connected part of correlations, $\left\langle n n^{\prime}\right\rangle_{\mathrm{c}} \equiv\left\langle n n^{\prime}\right\rangle-\rho^{2}$. For large enough systems $C(t)$ calculated in the propensity ensemble coincides with the standard autocorrelation function. A second interesting function is the cross-correlation or overlap $Q(t)$ between two different configurations at time $t$ in the propensity ensemble, $Q(t) \equiv\left\langle n_{i, \alpha}(t) n_{i, \beta}(t)\right\rangle_{\mathrm{c}} /\left\langle n_{i, \alpha}(0) n_{i, \beta}(0)\right\rangle_{\mathrm{c}}$, where $\alpha, \beta$ indicate different trajectories with identical initial conditions.

Figure 12(a) shows $C(t)$ and $Q(t)$ for the (2)-TLG. As expected, $C(t) \geq Q(t)$ for all $t$, i.e., the distance between initial and final configurations along a trajectory is always larger than that between two different final states. In fact, for any reversible dynamics $Q(t)=C(2 t)$ as long as the initial conditions are in equilibrium (for a discussion of the behaviour of $Q$ in aging see e.g. [15]). Interestingly, when $C(t)$ is much slower than an exponential, as in the present case where it appears to be logarithmic, the ratio $Q(t) / C(t)$ remains almost constant at long times. This is to be contrasted to the simple non-interacting lattice gas, where all decorrelation is through the diffusion of vacancies,
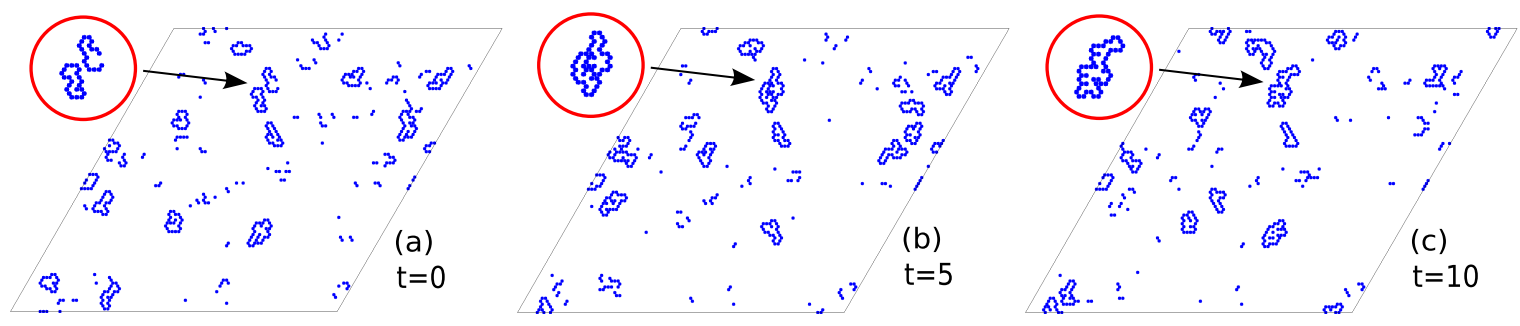

Figure 11. The motion of particles connected to the largest vacancy clusters [14. 

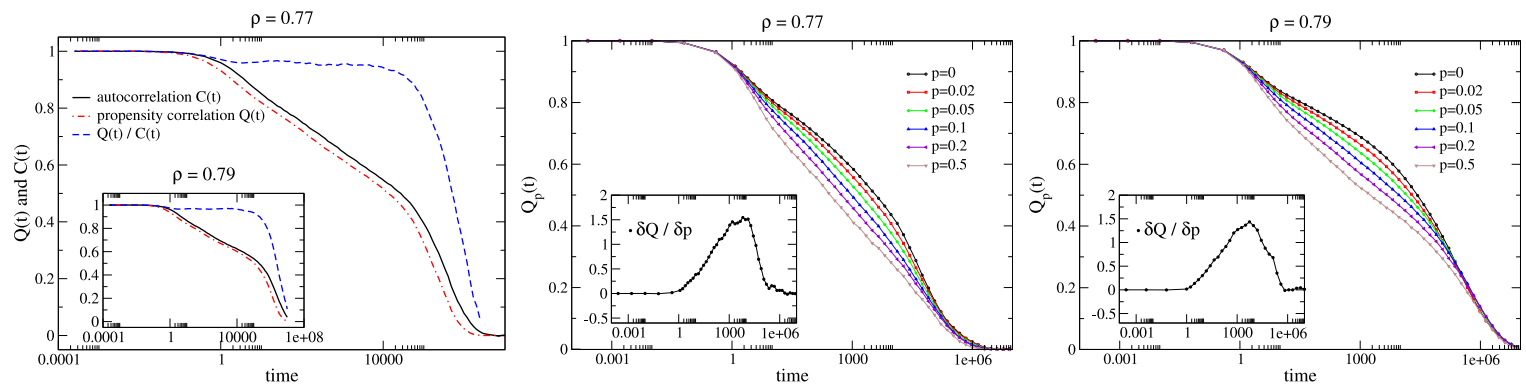

Figure 12. (a) Autocorrelation function $C(t)$ and propensity overlap $Q(t)$ in the (2)TLG for two different densities $\rho$ (main panel and inset). The dashed line is the ratio $Q(t) / C(t)$. (b) Overlaps $Q_{p}(t)$ for different values of $p$ at density $\rho=0.77$. The inset shows the response $\delta Q_{p}(t) /\left.\delta p\right|_{p=0}$. (c) Same as (b) but for density $\rho=0.79$.

and for which $Q(t) / C(t) \approx C(t)$.

The above illustrates how trajectories within the propensity ensemble are correlated through their common initial conditions. The sensitivity to the initial state can be tested by the response to perturbations in the initial conditions. Consider the case of two trajectories $\alpha$ and $\beta$ whose starting configurations differ only in the location of a fraction $p$ of their vacancies. We denote by $Q_{p}(t)$ their normalized overlap. In the case of a non-constrained lattice gas we have $Q_{p}(t)=Q_{p=0}(t)$, i.e., the initial distance is preserved in the trajectories at all times. Figs. 12(b) and (c) show the overlaps for the (2)-TLG. In this case the distance between configurations increases. The corresponding response, $\delta Q_{p}(t) /\left.\delta p\right|_{p=0}$, shown in the insets, is non-monotonic and peaks at times around the relaxation time.

As argued in [16, 17, sensitivity to initial conditions should be a signature of the dynamic phase transition which underlies dynamic heterogeneity in facilitated models in general: dynamics takes place very close to phase coexistence between an active phase, where motion is plentiful, and an inactive phase, where motion is scarce. Due to this phase equilibrium, initial conditions play an important role since they act as boundary fields in space-time. Dynamic propensity appears to be a useful tool to study their relevance.

\section{Acknowledgments}

We thank Peter Harrowell for correspondence. This work was supported by EPSRC grants GR/R83712/01, GR/S54074/01, and University of Nottingham grant FEF3024.

\section{References}

[1] For reviews see: M.D. Ediger, C.A. Angell and S.R. Nagel, J. Phys. Chem. 100, 13200 (1996); C.A. Angell, Science 267, 1924 (1995); P.G. Debenedetti and F.H. Stillinger, Nature 410, 259 (2001).

[2] For reviews see: H. Sillescu, J. Non-Cryst. Solids 243, 81 (1999); M.D. Ediger, Annu. Rev. Phys. Chem. 51, 99 (2000); S.C. Glotzer, J. Non-Cryst. Solids, 274, 342 (2000); R. Richert, J. Phys. 
Condens. Matter 14, R703 (2002); H. C. Andersen, Proc. Natl. Acad. Sci. U. S. A. 102, 6686 (2005).

[3] A. Widmer-Cooper, P. Harrowell and H. Fynewever, Phys. Rev. Lett. 93, 135701 (2004); A. Widmer-Cooper and P. Harrowell, J. Phys. Condens. Matter 17, S4025 (2005).

[4] A. Widmer-Cooper and P. Harrowell, Phys. Rev. Lett. 96, 185701 (2006).

[5] J. Jäckle and A. Krönig, J. Phys. Condens. Matter 6, 7633 (1994); 7655 (1994).

[6] W. Kob and H.C. Andersen, Phys. Rev. E 48, 4364 (1993).

[7] For recent results see, for example, J. Kurchan, L. Peliti and M. Sellito, Europhys. Lett. 39, 365 (1997); E. Marinari and E. Pitard, Euro. Phys. Lett. 69, 235 (2005); A. Lawlor et al., Phys. Rev. E 72, 21401 (2005).

[8] C. Toninelli, G. Biroli and D.S. Fisher, Phys. Rev. Lett. 92, 185504 (2004); C. Toninelli and G. Biroli, J. Stat. Phys. 117, 27 (2004).

[9] For a comprehensive review see: F. Ritort and P. Sollich, Adv. Phys. 52, 219 (2003).

[10] A.C. Pan, J.P. Garrahan and D. Chandler, Phys. Rev. E 72, 041106 (2005).

[11] M.E.J. Newman and G.T. Barkema, Monte Carlo Methods in Statistical Physics (Oxford University Press, Oxford, 1999).

[12] L. Berthier, D. Chandler and J.P. Garrahan, Europhys. Lett. 69, 320 (2005).

[13] J. Hoshen and R. Kopleman, Phys. Rev. B 14, 3438 (1976).

[14] These are snapshots from animations available from http://www.nottingham.ac.uk/ $\sim$ ppxloh/propensity.

[15] A. Barrat, R. Burioni and M. Mezard, J. Phys. A 29, 1311 (1996).

[16] M. Merolle, J.P. Garrahan and D. Chandler, Proc. Natl. Acad. Sci. USA 102, 10837 (2005).

[17] R.L. Jack, J.P. Garrahan and D. Chandler, J. Chem. Phys. to appear; cond-mat/0604068. 